\title{
Casimir Effect for a Massive Scalar Field Under Mixed Boundary Conditions
}

\author{
A.C. Aguiar Pinto, T.M. Britto, R. Bunchaft, F. Pascoal, and F.S.S. da Rosa \\ Universidade Federal do Rio de Janeiro, Instituto de Física, \\ Caixa Postal 68528, CEP.: 21945-970, Rio de Janeiro-RJ, Brazil
}

Received on 25 April, 2003. Revised version received on 2 September, 2003.

\begin{abstract}
We discuss the Casimir effect for a massive bosonic field with mixed (Dirichlet-Neumann) boundary conditions. We use the $\zeta$-function regularization prescription to obtain our physical results. Particularly, we analyse how the Casimir energy varies with the mass of the field and compare this mass dependence with those obtained for other boundary conditions. This is done graphically. Some other graphs involving a massive fermionic field are also included.
\end{abstract}

\section{Introduction}

Although the standard (electromagnetic) Casimir effect was proposed in 1948 by H.B.G Casimir[1], the Casimir effect in its general form ${ }^{1}$ is a very active area of research nowadays (for a detailed discussion of the effect and its applications see $[2,3,4]$ and references therein). This is due in part to its interdisciplinary character, once it is of some relevance not only in QED, but also in condensed matter physics, theories with compactified extra dimensions, gravitation and cosmology, mathematical physics and even in nanotechnology, on the construction of electromechanical devices.

Though the first experiment on Casimir effect has been performed by Sparnaay [5] in 1958 (with only a qualitative agreement), it was just recently that new measurements with slightly different setups were made with a high accuracy by many authors [6].

Although the Casimir effect disappears as the mass of the field goes to infinity, as expected (since in this limit there are no more quantum fluctuations), the precise way the Casimir energy varies as we change the mass is not obvious in some cases $[4,7]$.

Here we shall compute the influence of the mass in the Casimir energy of a bosonic field submitted to mixed boundary conditions (BC) and compare the obtained results with those for other $\mathrm{BC}$ already existent in the literature. Mixed $\mathrm{BC}$ are interesting mainly because they may provide repulsive Casimir forces. In fact, T.H. Boyer [8] studied the Casimir force for electromagnetic field between two parallel plates where one of them was a perfectly conductor plate and the other was an infinitely permeable one. This result can be mimicked by a massless scalar field submitted to mixed BC $[9,10]$. Nowadays, the study of repulsive Casimir forces has attracted the attention of physicists, since the existence of paramagnetic material with non-trivial permeability may exist [11], though there is some controversy about it $[12,13]$. Further, repulsive Casimir forces may be of some relevance in the construction of nanodevices where the attractive forces could lead to restrictions in these devices [4, 14]. Mixed $\mathrm{BC}$ have also been considered in the context of cavity QED [15-18].

This paper is organized as follows. In section 2, we calculate the Casimir energy for a massive bosonic field under mixed BC. In section 3, we compare the mass dependence of the Casimir energy of a bosonic field obtained in section 2 with those obtained with other BC, namely, Dirichlet, periodic and antiperiodic BC. This is done graphically. We also compare (graphically) the mass dependence of the Casimir energy of a fermionic field under distinct BC. However, in this case, we use only three $\mathrm{BC}$, namely, $\mathrm{MIT}^{2}$, periodic and antiperiodic BC, since there is no sense to impose Dirichlet or Neumann BC in the Dirac equation. We also make a comparison between the Casimir energy for a bosonic field under Dirichlet BC with a fermionic field under MIT BC. In section 4, we calculate the Casimir pressure for the massive scalar fields and plot some graphs. Last section is left for the final remarks and conclusions.

\section{Casimir energy for a massive scalar field with mixed boundary condi- tion}

Since massless scalar fields under Neumann or mixed BC may exhibt peculiar results regarding the signal of the Casimir force between the boundaries involved, it seems natural to investigate whether these results will persist for

\footnotetext{
${ }^{1}$ Every vacuum energy shift of any field (not necessarily the electromagnetic field) caused by the imposition of boundary conditions, dictated, for instance, by the presence of material surfaces, or even due to the compactification of some dimensions, is also refered as Casimir effect.

${ }^{2}$ The fermionic Casimir energy was first computed by Johnson[19] in the context of the MIT-bag model for a massless Dirac field confined between parallel planes with separation $a$
} 
a massive scalar field. Let us then consider a massive scalar field in a $d+1$ Euclidian spacetime subjected to a Dirichlet boundary condition at $x_{d}=0$ and a Neumann BC at $x_{d}=a$ :

$$
\begin{gathered}
\phi\left(\tau, x_{1}, x_{2}, \ldots, x_{d}=0\right)=0 \\
\frac{\partial \phi}{\partial x_{d}}\left(\tau, x_{1}, x_{2}, \ldots, x_{d}=a\right)=0 .
\end{gathered}
$$

Using the $\zeta$-function regularization prescription [20], the vacuum energy can be written as:

$$
\mathcal{E}_{\text {cas }}(a, m)=-\left.\frac{1}{2 T_{E}} \frac{\partial \zeta_{A}}{\partial s}\right|_{s=0},
$$

where

$$
\zeta_{A}(s)=\operatorname{tr} A^{-s}=\int d^{d+1} x\left\langle x\left|A^{-s}\right| x\right\rangle
$$

with $A=-\partial_{E}^{2}+m^{2}$. In Eq.(1), $T_{E}$ is the euclidean time and it is implicit an analytic continuation for the whole complex plane. Such conditions lead us to the following eigenvalues:

$$
\left\{\kappa^{2}+(n+1 / 2)^{2}(\pi / a)^{2} \mid \kappa^{2}=k_{1}^{2}+\ldots+k_{d}^{2} ; n \in \mathbb{N}\right\} .
$$

With these eigenvalues, we have:

$$
\begin{aligned}
\zeta_{A}(s) & =\frac{T_{E} L^{d-1}}{(2 \pi)^{d}} \sum_{n=0}^{\infty} \int d k_{1} \ldots d k_{d}\left\{\kappa^{2}+\left(n+\frac{1}{2}\right)^{2} \frac{\pi^{2}}{a^{2}}+m^{2}\right\}^{-s} \\
& =\frac{T_{E} L^{d-1}}{2^{d-1} \pi^{d / 2} \Gamma(d / 2)} \sum_{n=0}^{\infty} \int_{0}^{\infty} d \kappa \kappa^{2\left(\frac{d-1}{2}\right)}\left\{\kappa^{2}+\left(n+\frac{1}{2}\right)^{2} \frac{\pi^{2}}{a^{2}}+m^{2}\right\}^{-s},
\end{aligned}
$$

where $L^{d-1}$ is the area of the hiperplane and we used that $\oint d \Omega_{d}=\frac{2 \pi^{d / 2}}{\Gamma(d / 2)}$.

Using the following integral representation of the Euler beta function $B(x, y)=\frac{\Gamma(x) \Gamma(y)}{\Gamma(x+y)}$

$$
\int_{0}^{\infty} d x\left(x^{2}\right)^{\frac{\alpha-1}{2}}\left(x^{2}+A^{2}\right)^{\beta-1}=\frac{1}{2}\left(A^{2}\right)^{\frac{\alpha}{2}+\beta-1} B(\alpha / 2,1-\beta-\alpha / 2)
$$

we get

$$
\zeta_{A}(s)=\frac{T_{E} L^{d-1}}{2^{d-1} \pi^{d / 2}} \frac{\Gamma(s-d / 2)}{\Gamma(s)} \sum_{n=o d d} \frac{1}{2}\left\{\frac{n^{2} \pi^{2}}{4 a^{2}}+m^{2}\right\}^{\frac{d}{2}-s} .
$$

Introducing the Epstein function, defined as

$$
E_{1}^{M^{2}}(z, a)=\sum_{n=1}^{\infty} \frac{1}{\left(a n^{2}+M^{2}\right)^{z}},
$$

equation (6) can be cast into the form:

$$
\zeta_{A}(s)=\frac{T_{E} L^{d-1} \Gamma(s-d / 2)}{4^{d-s} \pi^{2 s-d / 2} \Gamma(s) a^{d-2 s}}\left[E_{1}^{(2 m a / \pi)^{2}}(s-d / 2 ; 1)-4^{d / 2-s} E_{1}^{(2 m a / \pi)^{2} / 4}(s-d / 2 ; 1)\right] .
$$

The last expression is not well defined for $\Re(s)>1 / 2$, so one can say it is useless for our purposes, since we need it at $s=0$. However, as it is well known in the literature [21], this function is suscetible to an analytic continuation into a meromorphic function in the whole complex plane, namely:

$$
\begin{aligned}
E_{1}^{M^{2}}(z, a) & =\frac{1}{2 M^{2 z}}+\left(\frac{\pi}{a}\right)^{1 / 2} \frac{1}{2 M^{2 z-1} \Gamma(z)} \times \\
& \times\left[\Gamma\left(z-\frac{1}{2}\right)+4 \sum_{n=1}^{\infty} \frac{a^{(1-2 z) / 4}}{(\pi M n)^{(1 / 2)-z}} K_{(1 / 2)-z}\left(\frac{2 \pi M n}{a^{1 / 2}}\right)\right],
\end{aligned}
$$

where $K_{\nu}(z)$ is the modified Bessel function of second type. We express then the generalized zeta function by the following equation

$$
\zeta_{A}(s)=\frac{T_{E} L^{d-1}}{2^{\frac{3 d}{2}-\frac{3}{2}-s} \pi^{\frac{d+1}{2}}} \frac{m^{\frac{d+1}{2}-s}}{a^{\frac{d-1}{2}+s}} \frac{1}{\Gamma(z)} \sum_{n=1}^{\infty} n^{s-\frac{d}{2}-\frac{1}{2}}\left[K_{s-\left(\frac{d+1}{2}\right)}(4 m n a)-\frac{1}{2^{s+\frac{1-d}{2}}} K_{s-\left(\frac{d+1}{2}\right)}(2 m n a)\right] .
$$


Computing the $s$ derivative at $s=0$ and using Eq.(1), we obtain our main result:

$$
\mathcal{E}_{c a s}(a, m)=-\frac{L^{d-1}}{2^{\frac{3 d-1}{2}} \pi^{\frac{d+1}{2}}} \frac{m^{\frac{d+1}{2}}}{a^{\frac{d-1}{2}}} \sum_{n=1}^{\infty} \frac{1}{n^{\frac{d+1}{2}}}\left[K_{-\frac{d+1}{2}}(4 m n a)-2^{\frac{d-1}{2}} K_{-\frac{d+1}{2}}(2 m n a)\right] .
$$

If $d=3$, for instance, one can easily obtain the limits for the last expression when $m a<<1$ or $m a>>1$, respectively:

$$
\mathcal{E}_{\text {cas }}(a, m) \approx \frac{7}{8} \frac{L^{2} \pi^{2}}{1440} \frac{1}{a^{3}}-\frac{1}{192} \frac{L^{2}}{a} m^{2} \quad(m a<<1)
$$

and

$$
\mathcal{E}_{\text {cas }}(a, m) \approx \frac{L^{2}}{16}\left(\frac{m}{\pi a}\right)^{3 / 2} e^{-2 m a} \quad(m a>>1)
$$

In eq.(13), we see that the Casimir energy for two parallel plates vanishes exponentially as the mass goes to infinity (for spherical geometries it vanishes as a negative power of the mass [4]). On the other hand, for a small mass, we have the well known result for the corresponding massless case (first term of r.h.s. stands for $-\frac{7}{8}$ times the standard result of the Casimir energy for a massless scalar field subjected to Dirichlet BC) $[8,10]$ plus a small (negative) correction in $m^{2}$.

\section{The influence of the mass in the Casimir effect: Graphical results}

In this section we shall compare the result (11) obtained in the previous section with those already existent in the literature obtained with other boundary conditions, namely, Dirichlet (D), periodic (P) and antiperiodic (A) ones. In order to make such a comparison, it is convenient to write down explicitly the respective expressions for the Casimir energies for these cases:

$$
\begin{aligned}
\mathcal{E}_{c a s}^{D}(a, m)= & -\frac{2 L^{d-1}}{(4 \pi)^{(d+1) / 2}} \frac{m^{(d+1) / 2}}{a^{(d-1) / 2}} \sum_{n=1}^{\infty} n^{-(d+1) / 2} K_{-(d+1) / 2}(2 m n a) ; \\
\mathcal{E}_{c a s}^{P}(a, m)= & -\frac{L^{d-1}}{2^{(d-1) / 2} \pi^{(d+1) / 2}} \frac{m^{(d+1) / 2}}{a^{(d-1) / 2}} \sum_{n=1}^{\infty} n^{-(d+1) / 2} K_{-(d+1) / 2}(m n a) ; \\
\mathcal{E}_{c a s}^{A}(a, m)= & -\frac{L^{d-1}}{2^{d-1} \pi^{(d+1) / 2}} \frac{m^{(d+1) / 2}}{a^{(d-1) / 2}} \sum_{n=1}^{\infty} n^{-(d+1) / 2}\left[K_{-(d+1) / 2}(2 m n a)-\right. \\
& \left.-2^{(d-1) / 2} K_{-(d+1) / 2}(m n a)\right] .
\end{aligned}
$$

The first important thing to be noticed is the simple relation that arises between the Casimir energies under mixed BC and Dirichlet one:

$$
\mathcal{E}_{\text {cas }}^{M}(a, m)=\mathcal{E}_{\text {cas }}^{D}(2 a, m)-\mathcal{E}_{\text {cas }}^{D}(a, m) .
$$

For the zero mass limit in $d$ spatial dimensions the previous equation leads to

$$
\begin{aligned}
\mathcal{E}_{\text {cas }}^{M}(a, 0) & =\mathcal{E}_{\text {cas }}^{D}(2 a, 0)-\mathcal{E}_{\text {cas }}^{D}(a, 0) \\
& =\left(\frac{1}{2^{d}}-1\right) \mathcal{E}_{\text {cas }}^{D}(a, 0)
\end{aligned}
$$

where we used the fact that, for $m=0$, the Casimir energies for such setups are proportional to $1 / \ell^{d}$, with $\ell$ being the distance between the planes. This gives the well known $-7 / 8$ factor for the particular case where $d=3$ already mentioned.

Also, it is straightforward to verify that the antiperiodicperiodic comparison mimics this behaviour. In other words, we may also write

$$
\mathcal{E}_{\text {cas }}^{A}(a, m)=\mathcal{E}_{\text {cas }}^{P}(2 a, m)-\mathcal{E}_{\text {cas }}^{P}(a, m) .
$$

These "duality" relations between the Casimir energies under different BC, given by equations (17) and (19), can be formally established by a simple heuristic argument. Let us consider, for instance, the Dirichlet-mixed case. Casimir energy for the mixed $\mathrm{BC}$ can be written as 


$$
\begin{aligned}
\mathcal{E}_{\text {cas }}^{M}(a, m) & =N \int d k_{1} \cdots d k_{d} \sum_{n=0}^{\infty} \sqrt{(2 n+1)^{2}\left(\frac{\pi}{2 a}\right)^{2}+\kappa^{2}+m^{2}} \\
& =N \int d k_{1} \cdots d k_{d} \sum_{n=\text { odd }}^{\infty} \sqrt{n^{2}\left(\frac{\pi}{2 a}\right)^{2}+\kappa^{2}+m^{2}}
\end{aligned}
$$

where $N$ is a constant and $\kappa=k_{1} \cdots k_{d}$. Now, let us use the simple trick of adding and subtracting the sum over even $n$ $\left(n=2 n^{\prime}\right)$, that is

$$
\begin{aligned}
\mathcal{E}_{\text {cas }}^{M}(a, m) & =N \int d k_{1} \cdots d k_{d} \sum_{n=1}^{\infty} \sqrt{n^{2}\left(\frac{\pi}{2 a}\right)^{2}+\kappa^{2}+m^{2}}- \\
& -N \int d k_{1} \cdots d k_{d} \sum_{n^{\prime}=1}^{\infty} \sqrt{\left(2 n^{\prime}\right)^{2}\left(\frac{\pi}{2 a}\right)^{2}+\kappa^{2}+m^{2}} \\
& =\mathcal{E}_{\text {cas }}^{D}(2 a, m)-\mathcal{E}_{\text {cas }}^{D}(a, m),
\end{aligned}
$$

where we identify the first term of the r.h.s. of (21) as the Casimir energy for a massive scalar field under Dirichlet BC with distance $2 a$ between the planes and the second term as the same Casimir energy with distance $a$.

Considering now the normalized energy, which is defined as

$$
\tilde{E}(m, a)=\frac{\mathcal{E}_{\text {cas }}(m, a)}{\mathcal{E}_{\text {cas }}(0, a)}
$$

one can easily show that

$$
\begin{gathered}
\tilde{E}^{A}(2 m a)=\tilde{E}^{P}(m a) \\
\tilde{E}^{M}(2 m a)=\tilde{E}^{D}(m a) .
\end{gathered}
$$

Note that the normalized energies depend on the mass and the distance only through the product ma. Using Eqs.(11)(16) and (22), we plot our first graph for the normalized energies with $d=3$ (see Fig. 1). As already mentioned, note that in all graphs the energy vanishes as the mass goes to infinity.

For a massive fermionic field in $d=3$, we may plot the following graph:

In the fermionic case, the Casimir energies for periodic and antiperiodic boundary conditions are equal to a quarter of the bosonic Casimir energy with the same $\mathrm{BC}$ in $d=3$. The Casimir energy with MIT boundary condition for a massive fermionic field was first computed by Mamaev and Trunov [22] (see also Ref.[3]), but can be cast into the form [23]:

$$
\begin{gathered}
\mathcal{E}_{\text {cas }}^{M I T}(a)=-\frac{L^{d-1} \alpha(d) \mu^{(d-2) / 2}}{2^{d+1} \pi^{d / 2} \Gamma(d / 2) a^{d}} \sum_{k=1}^{\infty} \frac{(-1)^{k+1}}{k^{d / 2+2}} \\
\left.\Gamma\left(\frac{d}{2}+k\right) \frac{d}{d \lambda}\left[\lambda^{-d / 2} W_{-k, \frac{d-1}{2}}(4 \mu k \lambda)\right]\right|_{\lambda=1},
\end{gathered}
$$

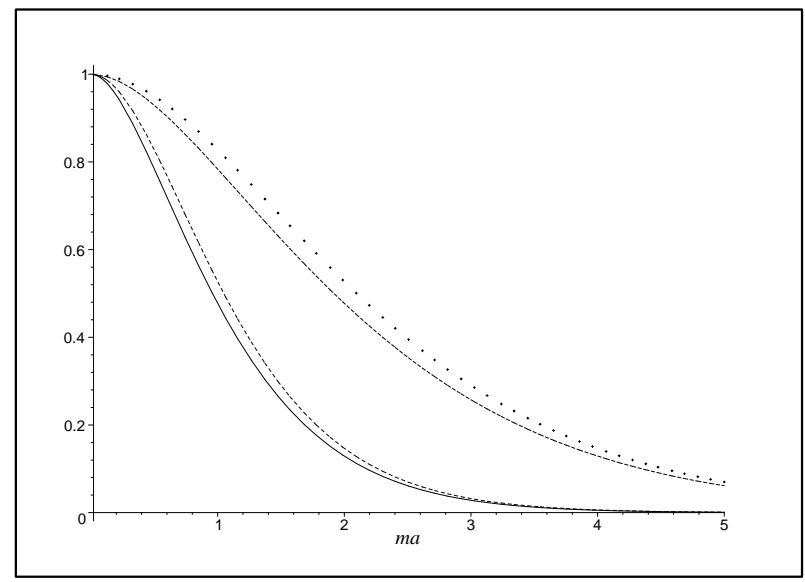

Figure 1. The vertical axis represents the normalized Casimir energy for a massive field. In the graph, we have the following BC (from top to bottom): antiperiodic, periodic, mixed and Dirichlet respectively.

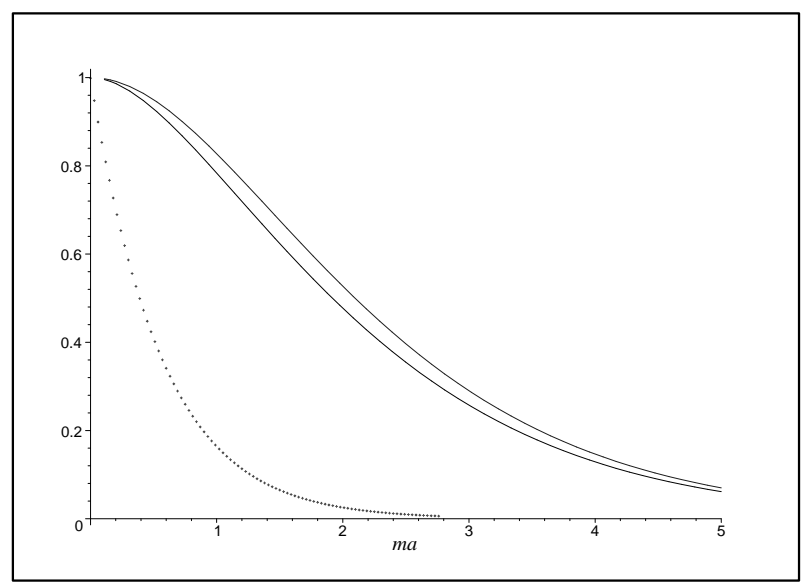

Figure 2. Massive fermionic field with three BC (from top to bottom): antiperiodic, periodic and MIT.

where $\mu:=m a, W_{\nu, \mu}(z)$ is the Whittaker function [24] and $\alpha(d)$ is a dimensionless factor that counts the number of dif- 
ferent spin states and is given by $2^{(d-1) / 2}$, for $d$ odd, and by $2^{(d-2) / 2}$ if $d$ is even.

Once the Dirichlet and MIT boundary conditions are the most natural ones for the bosonic and fermionic fields respectively, we also compare graphically the Casimir energy for these two cases (see Fig. 3). It is worth emphasizing that the fermionic Casimir energy with MIT boundary condition goes to zero faster, as the mass of the field increases, than the Casimir energy for a massive bosonic field with Dirichlet boundary condition.

\section{The Casimir pressure of massive scalar fields}

One interesting feature of the Casimir effect is that eventhough it is a genuine quantum effect, it predicts macroscopic effects, as an attractive force between two uncharged parallel perfectly conducting plates [1]. One can obtain the force per unit area (Casimir pressure) through the relation

$$
\mathcal{F}(m, a)=-\frac{\partial \mathcal{E}(m, a)}{\partial a}
$$

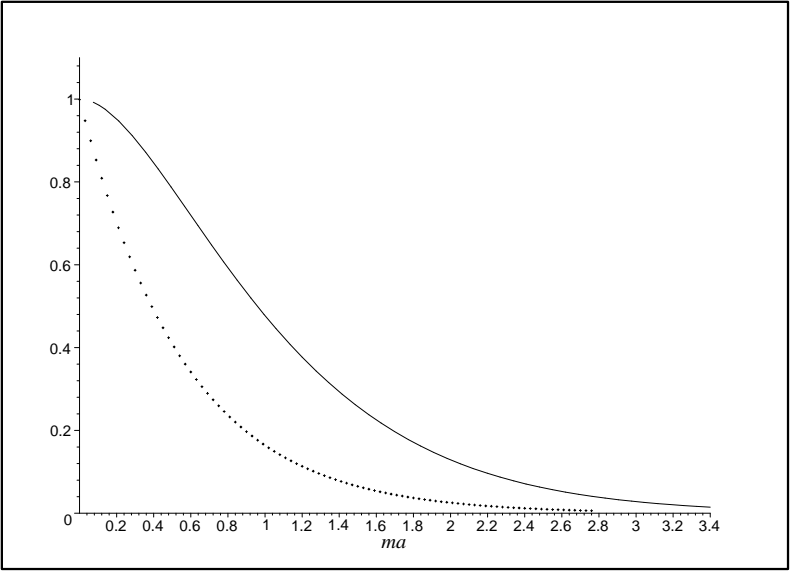

Figure 3. From top to bottom we have the Casimir energies for a massive bosonic field subjected to Dirichlet $\mathrm{BC}$ and for a massive fermionic field submitted to MIT BC, respectively.

where $\mathcal{E}(m, a)=\mathcal{E}_{\text {cas }}(m, a) / L^{d-1}$. Using Eq.(26), we are able to extract the Casimir pressure for scalar fields when subjected to mixed boundary conditions, which is (for the case $d=3$ )

$$
\begin{aligned}
\mathcal{F}_{M}(m, a) & =-\frac{m^{2}}{16 a^{2} \pi^{2}}\left[\sum_{n=1}^{\infty} \frac{3}{n^{2}} K_{2}(4 \text { man })+\sum_{n=1}^{\infty} \frac{4 m a}{n} K_{1}(4 m a n)\right]+ \\
& +\frac{m^{2}}{8 a^{2} \pi^{2}}\left[\sum_{n=1}^{\infty} \frac{3}{n^{2}} K_{2}(2 \text { man })+\sum_{n=1}^{\infty} \frac{2 m a}{n} K_{1}(2 m a n)\right] .
\end{aligned}
$$

The explicit formulas for the other boundary conditions are

$$
\begin{gathered}
\mathcal{F}_{D}(m, a)=-\frac{m^{2}}{8 a^{2} \pi^{2}}\left[\sum_{n=1}^{\infty} \frac{3}{n^{2}} K_{2}(2 \operatorname{man})+\sum_{n=1}^{\infty} \frac{2 m a}{n} K_{1}(2 m a n)\right] \\
\mathcal{F}_{P}(m, a)=-\frac{m^{2}}{2 a^{2} \pi^{2}}\left[\sum_{n=1}^{\infty} \frac{3}{n^{2}} K_{2}(\text { man })+\sum_{n=1}^{\infty} \frac{m a}{n} K_{1}(\text { man })\right]
\end{gathered}
$$

and

$$
\begin{array}{r}
\mathcal{F}_{A}(m, a)=-\frac{m^{2}}{4 a^{2} \pi^{2}}\left[\sum_{n=1}^{\infty} \frac{3}{n^{2}} K_{2}(2 \operatorname{man})+\sum_{n=1}^{\infty} \frac{2 m a}{n} K_{1}(2 \operatorname{man})\right]+ \\
+\frac{m^{2}}{2 a^{2} \pi^{2}}\left[\sum_{n=1}^{\infty} \frac{3}{n^{2}} K_{2}(\operatorname{man})+\sum_{n=1}^{\infty} \frac{m a}{n} K_{1}(\operatorname{man})\right] .
\end{array}
$$

We may show now the comparative plot between the different pressures (their modules, actually) 


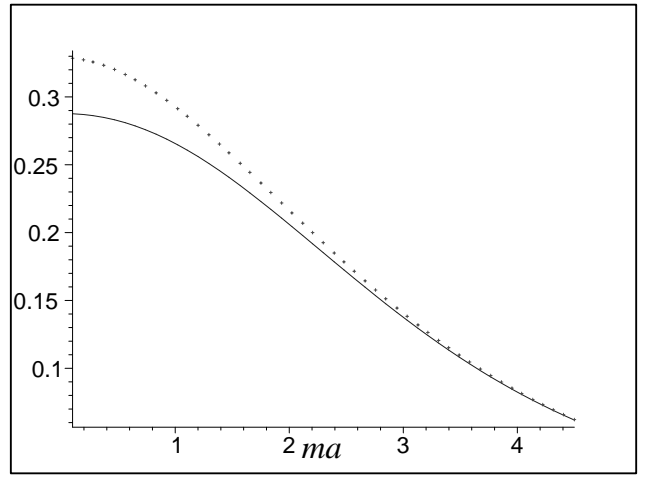

Figure 4. In this graphic: we plot the modules of pressures with antiperiodic BC (line) and periodic BC (point).

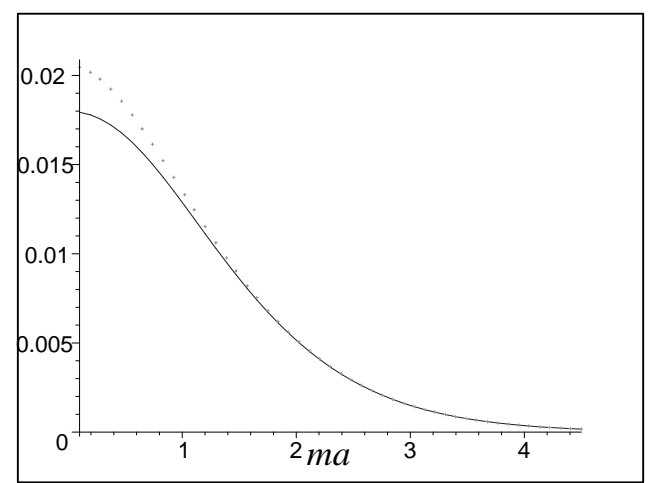

Figure 5. In this graphic we plot the modules of pressures with mixed BC (line) and Dirichlet BC (point).

which is not very different from the previous plot, comparing normalized energies. Using Eqs.(27)-(30), one can obtain analogous relations to (17) and (19) (see also Eq.(26))

$$
\mathcal{F}_{M}(m, a)=2 \mathcal{F}_{D}(m, 2 a)-\mathcal{F}_{D}(m, a)
$$

and

$$
\mathcal{F}_{A}(m, a)=2 \mathcal{F}_{P}(m, 2 a)-\mathcal{F}_{P}(m, a)
$$

\section{Conclusions and final remarks}

In this work we computed the Casimir energy and the Casimir pressure for a massive scalar field submitted to mixed boundary conditions. Our physical results were obtained with the aid of the generalized $\zeta$-function regularization prescription, but other methods could be used as well. The limits of small and large mass were taken and are in agreement with expected results. In fact, the zero mass limit coincides precisely with the results found in literature $[8,10]$ and the first correction in the mass has opposite sign compared with the zero mass case. This means that the first correction weakens the Casimir energy.

For finite mass, we compared our result with those obtained from the literature for other boundary conditions and showed that the behaviour of the Casimir energy with mixed $\mathrm{BC}$ is analogous to the others (the Casimir energy is a monotonically decrescent function of $m a$ ).
As can be observed in Fig. 1, the relation between the Dirichlet BC and mixed BC is completely analogous to that between the periodic and antiperiodic BC. This can be easily understood from Eqs. (17) and (19).

We have also presented a graph involving a massive fermionic field under different $\mathrm{BC}$ which shows that the Casimir energy with MIT BC diminishes much more rapidly than the Casimir energy with periodic or antiperiodic BC. It is worth emphasizing that, when compared with the Casimir energy for a massive scalar field under Dirichlet BC, the fermionic Casimir energy with MIT BC also is much smaller for a given value of $m a$.

Though the influence of the mass in the Casimir energies for the cases discussed here were of considerable simplicity, this will not be the case for more involved situations, with other geometries and non trivial topologies, but that will be left for future works.

\section{Acknowledgments}

The authors would like to thank C. Farina for valuable comments. T.M.B., F.P. and R.B. would like to thank CAPES for financial support. F.S.S.R. and A.C.A.P. acknowledge $\mathrm{CNPq}$ for financial support.

\section{References}

[1] H.B.G. Casimir, Proc. K. Ned. Akad. Wet. 51, 793 (1948).

[2] G. Plunien, B. Muller, and W. Greiner, Phys. Rep. 134, 89 (1986).

[3] V.M. Mostepanenko and N.N. Trunov, The Casimir Effect and Its Applications, (Clarendon Press, Oxford, 1997).

[4] M. Bordag, U. Mohideen, and V.M. Mostepanenko, Phys. Rep. 353, 205 (2001).

[5] M.J. Sparnaay, Physica 24, 751 (1958).

[6] S.K. Lamoreaux, Phys. Rev. Lett. 79, 5 (1997);

U. Mohideen and A. Roy, Phys. Rev. Lett. 81, 4549 (1998);

A. Roy and U. Mohideen, Phys. Rev. Lett. 82, 4380 (1999);

A. Roy, C.-Y. Lin and U. Mohideen, Phys. Rev. D 60, 111101(R) (1999);

B.W. Harris, F. Chen, and U. Mohideen, Phys. Rev. A 62, 052109 (2000);

T. Ederth, Phys. Rev. A 62, 062104 (2000);

H.B. Chan, V.A. Aksyuk, R.N. Kleiman, D.J. Bishop, and F. Capasso, Science 291, 1941 (2001);

H.B. Chan, V.A. Aksyuk, R.N. Kleiman, D.J. Bishop, and F. Capasso, Phys. Rev. Lett. 87, 211801 (2001);

G. Bressi, G. Carugno, R. Onofrio, and G. Ruoso, Phys. Rev. Lett. 88, 041804 (2002).

[7] K. Kirsten, Spectral Functions in Mathematics and Physics, (Chapman \& Hall, Dordrecht - Netherlands, 2001).

[8] T.H. Boyer, Phys. Rev A 9, 2078 (1974).

[9] V. Hushwater, Am. J. Phys. 65, 381 (1997).

[10] M.V. Cougo-Pinto, C. Farina, and A. Tenório, Braz. J. Phys. 29, 371 (1999).

[11] O. Kenneth, I. Klich, and M. Revzen, Phys. Rev. D 65 , 045005 (2002);

O. Kenneth, I. Klich, A. Mann, and M. Revzen, Phys. Rev. Lett. 89, 033001 (2002). 
[12] D. Iannuzzi and F. Capasso, Phys. Rev. Lett. 91, 029101 (2003).

[13] O. Kenneth, I. Klich, A. Mann, and M. Revzen, Phys. Rev. Lett. 91, 029101 (2003).

[14] E. Buks and M.L. Roukes, Phys. Rev. B 63, 033402 (2001).

[15] M.V. Cougo-Pinto, C. Farina, F. Santos, and A. Tort, Phys. Lett. B 446, 170 (1999).

[16] M.V. Cougo-Pinto, C. Farina, F. Santos, and A. Tort, J. Phys. A 32, 4463 (1999).

[17] D.T. Alves, C. Farina, and A.C. Tort, Phys. Rev. A 61, 034102 (2000).

[18] D.T. Alves, F.A. Barone, C. Farina, and A.C. Tort, Phys. Rev. A 67, 022103 (2003).
[19] K. Johnson, Acta Phys. Polonica B 6, 865 (1975).

[20] E. Elizalde, S. D. Odintsov, A. Romeo, A. A. Bitsenko, and S. Zerbini, Zeta Regularization Techniques with Applications, (World Scientific, Singapore, 1994).

[21] E. Elizalde and A. Romeo, J. Math. Phys. 30, 1133 (1989);

E. Elizalde and A. Romeo, J. Math. Phys. 31, 771(E) (1990); K. Kirsten, J. Math. Phys. 35, 459 (1994).

[22] S.G. Mamaev and N.N. Trunov, Sov. Phys. 23, 551 (1980).

[23] E. Elizalde, F.C. Santos, and A.C. Tort, Int. J. Mod. Phys. A 18, 1761 (2003).

[24] E.T. Whittaker and G.N. Watson, A Course in Modern Analysis, $4^{\text {th }}$ ed. (Cambridge University Press, Cambridge, 1990). 